\title{
Presynaptic Ryanodine Receptor-Activated Calmodulin Kinase II Increases Vesicle Mobility and Potentiates Neuropeptide Release
}

\author{
Dinara Shakiryanova, ${ }^{1}$ Markus K. Klose, ${ }^{2}$ Yi Zhou, ${ }^{1}$ Tingting Gu, ${ }^{3}$ David L. Deitcher, ${ }^{4}$ Harold L. Atwood, ${ }^{2}$ \\ Randall S. Hewes, ${ }^{3}$ and Edwin S. Levitan ${ }^{1}$ \\ ${ }^{1}$ Department of Pharmacology, University of Pittsburgh, Pittsburgh, Pennsylvania 15261, 2Department of Physiology, University of Toronto, Toronto, \\ Ontario, Canada M5S 1A8, ${ }^{3}$ Departments of Zoology and Cell Biology, University of Oklahoma, Norman, Oklahoma 73019, and ${ }^{4}$ Department of \\ Neurobiology and Behavior, Cornell University, Ithaca, New York 14853
}

\begin{abstract}
Although it has been postulated that vesicle mobility is increased to enhance release of transmitters and neuropeptides, the mechanism responsible for increasing vesicle motion in nerve terminals and the effect of perturbing this mobilization on synaptic plasticity are unknown. Here, green fluorescent protein-tagged dense-core vesicles (DCVs) are imaged in Drosophila motor neuron terminals, where DCV mobility is increased for minutes after seconds of activity. $\mathrm{Ca}^{2+}$-induced $\mathrm{Ca}^{2+}$ release from presynaptic endoplasmic reticulum (ER) is shown to be necessary and sufficient for sustained DCV mobilization. However, this ryanodine receptor (RyR)-mediated effect is short-lived and only initiates signaling. Calmodulin kinase II (CaMKII), which is not activated directly by external Ca ${ }^{2+}$ influx, then acts as a downstream effector of released $\mathrm{ER} \mathrm{Ca}^{2+}$. RyR and CaMKII are essential for post-tetanic potentiation of neuropeptide secretion. Therefore, the presynaptic signaling pathway for increasing DCV mobility is identified and shown to be required for synaptic plasticity.
\end{abstract}

Key words: vesicle mobility; mobilization; dense-core vesicle; neuropeptide release; synaptic plasticity; Drosophila

\section{Introduction}

It has long been speculated that vesicle motion at synapses could be controlled to influence neurotransmission, but activitydependent increases in vesicle mobility (i.e., mobilization) in nerve terminals were detected directly only recently. This was first accomplished with neuropeptide-containing dense-core vesicles (DCVs) in Drosophila neuromuscular junction (NMJ) by using two methods, fluorescence recovery after photobleaching (FRAP) and image correlation analysis of time-lapse movies (Shakiryanova et al., 2005) [for movies and technical details, see Levitan et al. (2007)]. Subsequently, stimulus-induced vesicle motion was detected with mammalian neuroendocrine DCVs and frog NMJ small synaptic vesicles (SSVs) (Allersma et al., 2006; Gaffield et al., 2006). The increase in vesicle mobility in these diverse preparations shares common features. First, mobilized vesicles move randomly, in accordance with single particletracking studies that showed that releasable and reserve secretory vesicles move by diffusion, albeit at different rates (Han et al., 1999; Ng et al., 2003). Second, stimulation-induced mobility is unaffected by depolymerizing F-actin. Along with other experiments (Silverman et al., 2005; Gaffield et al., 2006; Tokuoka and

Received April 25, 2007; revised June 13, 2007; accepted June 13, 2007.

This work was supported by National Institutes of Health Grant NS32385 (E.S.L.) and by a research grant from the Canadian Institutes of Health Research (H.L.A.). We thank Chandra Ziegler for technical assistance.

Correspondence should be addressed to Dr. Edwin S. Levitan at the above address. E-mail: levitan@server.pharm.pitt.edu.

D0I:10.1523/JNEUROSCI.1879-07.2007

Copyright $\odot 2007$ Society for Neuroscience $\quad$ 0270-6474/07/277799-08\$15.00/0
Goda, 2006), this finding suggests that the long-standing question of whether F-actin is a barrier for vesicle immobilization or a track for motor-mediated translocation (Nunes et al., 2006; Scalettar, 2006) is not central to understanding stimulation-induced vesicle motion. Finally, the stimuli in each case are associated with $\mathrm{Ca}^{2+}$ influx, which was explicitly demonstrated to be required for enhancing vesicle mobility in two of the studies (Shakiryanova et al., 2005; Allersma et al., 2006). Although SSV mobilization is not seen in all neurons (Rea et al. 2004; Lemke and Klingauf, 2005), these shared properties indicate that a conserved mechanism may underlie mobilization of both SSVs and DCVs at sites that are capable of this regulation.

Mobilization of neuropeptide-containing DCVs likely involves sustained signal transduction because seconds of activity in Drosophila NMJ type Ib boutons induce many minutes of increased DCV mobility even after release is inhibited (Shakiryanova et al., 2005). However, the specific pathway activated by $\mathrm{Ca}^{2+}$ influx has not been identified. Thus, the role of this signaling pathway in synaptic function and plasticity is not known. Indeed, although pharmacological manipulations have been shown to produce DCV mobility increases that can account for increased release in vitro ( $\mathrm{Ng}$ et al., 2002, 2003), inhibiting physiological vesicle mobilization has never been shown to block potentiation of release in vivo.

Here, in vivo imaging of a green fluorescent protein (GFP)labeled neuropeptide, which reports native neuropeptide secretion (Heifetz and Wolfner, 2004; Husain and Ewer, 2004; Kula et al., 2006), shows that ryanodine receptor (RyR)-mediated $\mathrm{Ca}^{2+}$ 
release from presynaptic endoplasmic reticulum (ER) is necessary and sufficient for triggering DCV mobilization in the larval NMJ. Calmodulin kinase II (CaMKII) then acts as an effector of released ER $\mathrm{Ca}^{2+}$. Finally, RyR-mediated $\mathrm{Ca}^{2+}$ release and CaMKII are shown to be required for post-tetanic potentiation (PTP) of neuropeptide secretion.

\section{Materials and Methods}

Animals and preparations. Most experiments were performed on the third instar larval NMJ from transgenic Drosophila expressing Emerald GFP-tagged atrial natriuretic factor (elav-GAL4 UAS-preproANF-EMD) as described previously (Rao et al., 2001; Shakiryanova et al., 2005; Levitan et al., 2007). The temperature-sensitive dSERCA (Drosophila Sarco/ endoplasmic reticulum $\mathrm{Ca}^{2+}$ ATPase) mutant $\mathrm{Ca}-P 60 A^{\text {Kum } 170}$ (Kum170) and the targetable conditional dominant-negative UAS-

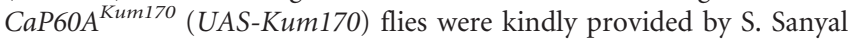
(Emory University, Atlanta, GA). In both cases, the $\mathrm{Ca}-\mathrm{P} 60$ refers to the P-type $\mathrm{Ca}^{2+}$-ATPase gene at chromosomal position $60 \mathrm{~A}$ on the right arm of the second chromosome, which is the sole SERCA gene in the Drosophila genome (Sanyal et al., 2005). For global disruption of SERCA, we studied larvae with the genotype elav-GAL4 UAS-preproANF-EMD; Kum170/CyO. For disruption of SERCA function only in neurons, we studied larvae with the genotype elav-GAL4 UAS-preproANF-EMD; $U A S-K u m 170 / C y O$, Act-GFP. The fluorescence signal from the balancer chromosome (i.e., produced by Act-GFP) is not localized to the NMJ and so did not interfere with synaptic bouton measurements. Furthermore, the use of a single pan-neuronal GAL4 driver and GAL4-responsive UAS constructs ensured that dominant-negative SERCA and the GFP-tagged peptide were coexpressed in neurons but not in postsynaptic muscle. To disable SERCA function in Kum170 and UAS-Kum170 lines, wanderingstage third instar larvae were placed in a chamber with $\mathrm{Ca}^{2+}$-free HL3 (see below) at $40^{\circ} \mathrm{C}$ for $8 \mathrm{~min}$. The animals were then filleted, and the solution was replaced as needed. UAS-Cameleon 2.1 flies (Diegelmann et al., 2002) were obtained from the Bloomington Stock Center (Bloomington, IN) and crossed with 386-GAL4 to induce expression in motor neurons.

Solutions. HL3 saline contained (in mM) $70 \mathrm{NaCl}, 5 \mathrm{KCl}, 1.5 \mathrm{CaCl}_{2}, 20$ $\mathrm{MgCl}_{2}, 10 \mathrm{NaHCO}_{3}, 5$ trehalose, 115 sucrose, and 5 sodium HEPES, $\mathrm{pH}$ 7.2. Calcium was substituted with $0.5 \mathrm{~mm}$ EGTA to generate $\mathrm{Ca}^{2+}$-free saline.

Electrical stimulation. Synaptic boutons were electrically stimulated via intact segmental motor nerves with a suction electrode. To minimize the muscle contractions, which disrupt imaging during stimulation, postsynaptic glutamate receptors were desensitized with $10 \mathrm{~mm}$ L-glutamate or blocked by including an antagonist, $100 \mu \mathrm{M}$ 1-napthylacetyl spermine trihydrochloride. Furthermore, the high$\mathrm{Mg} / \mathrm{Ca}$ HL3 solution was used for extracellular medium (see above). Finally, spontaneous muscle contractions were also reduced by cutting the ventral ganglion, which prevents input from a central pattern generator to motor neurons.

Imaging and data analysis. Imaging was performed with type Ib synaptic boutons on muscles 6 and 7, which contain $\sim 260$ DCVs (Shakiryanova et al., 2006), as described previously (Shakiryanova et al., 2005; Levitan et al., 2007). In brief, data were acquired with cooled CCD cameras, direct water-immersion $60 \times$ objectives, and wide-field epifluorescence microscopes. Although activity-induced DCV mobility changes in Drosophila NMJ are evident in time-lapse movies (Levitan et al., 2007), simple inspection is not quantitative. Therefore, mobility was quantified based on changes in the pixel-by-pixel correlation coefficients (CCs) between consecutive images in a time-lapse series. The CC was calculated with an ImageJ plug-in CC calc.java, which was written by A. Tully and is available at http://shell.abtech.org/ tully/ImageJ/index.html. For each experimental condition (e.g., control), nine images were acquired. After discarding out-of-focus images, the CC was calculated between sequential images, and a mean was determined for the data set. Image correlation spectroscopy uses such data to determine diffusion coefficients, but this depends on the assumption that moving particles are small compared with the volume sampled by a pixel on the camera chip. Because
DCVs are large and produce complex images in wide-field microscopy, this theory does not apply here. Therefore, as in a past study (Shakiryanova et al., 2005), we calculated the mobility index as 1-CC, which increases with mobility (i.e., the $\mathrm{CC}$ will be 1 when image pairs are identical and will drop when there is detectable DCV motion). This correlation assay allows for rapid and continual monitoring of reversible changes in mobility without significant photodamage (Shakiryanova et al., 2005; Levitan et al., 2007). Indeed, this approach could be used between spontaneous centrally generated repetitive muscle contractions in standard saline to detect DCV mobility increases that are larger [i.e., the mobility index reaches 0.2 (Shakiryanova et al., 2005)] than the responses to a single $15 \mathrm{~s}$ tetanus in HL3. Mobilization of Drosophila DCVs is also evident with FRAP. However, each FRAP assay takes minutes, and so this approach has poor time resolution. Furthermore, several bouts of photobleaching (each associated with photodamage) would be required to deduce the overall kinetics of mobilization with FRAP. Finally, interpretation of FRAP data are difficult with DCVs: the immobile fraction revealed by FRAP does not correspond to an immobile pool of DCVs but instead is caused by the unusually broad and non-Gaussian distribution of DCV diffusion coefficients (Ng et al., 2003). The goal here was to identify signaling required for DCV mobilization and to test whether this signaling affects release. Correlation analysis allows for reproducible and quantitative dynamic detection of changes in DCV mobility and so is well suited for this purpose.

Peptide secretion was measured as the loss of GFP fluorescence. Ratiometric $\mathrm{Ca}^{2+}$ imaging with Cameleon was performed with a 440DF20 excitation filter, a 455DRLP dichroic mirror, and alternating emission filters [480DF30 for enhanced cyan fluorescent protein (ECFP), 535DF25 for enhanced yellow fluorescent protein (EYFP)] on a filter wheel. The ratio of the emissions (EYFP/ECFP) was then determined. However, because the absolute value of this ratio depends on the specific optics and camera used, data were normalized to the initial ratio before stimulation and percentage changes from this ratio were calculated. For single wavelength $\mathrm{Ca}^{2+}$ imaging, cut motor nerves in Canton S larvae were loaded with dextran-conjugated Oregon Green Bapta-1 (OGB-1) and assayed as described previously (Macleod et al., 2002). In vivo caffeine responses were corrected for $\sim 5 \%$ quenching of OGB-1 detected with in vitro experiments (data not shown). Data from two to four boutons were pooled for each OGB-1 measurement. However, all other measurements were made from a single bouton in each experiment.

Reagents. Caffeine, veratridine, and 1-napthylacetyl spermine trihydrochloride were purchased from Sigma (St. Louis, MO). 4-Bromocalcimycin (4-Br-A13287) was purchased from Alexis Biochemicals (San Diego, CA). Cyclopiazonic acid (CPA) and thapsigargin (Tg) were obtained from Alomone Labs (Jerusalem, Israel). KN-92, KN-93, KN-62, ryanodine (high purity), Antennapedia autocamtide-2-related inhibitory peptide II (AIP-II), and Drosophila Antennapedia homeodomain (DT-5) were obtained from Calbiochem (La Jolla, CA). Stock solutions of Tg, CPA, veratridine, and KN-92 were prepared in DMSO. The final DMSO concentration in saline was no more than $0.1 \%$.

\section{Results \\ $\mathrm{Ca}^{2+}$ release from internal $\mathrm{Ca}^{2+}$ stores induces vesicle mobilization}

To test whether $\mathrm{Ca}^{2+}$ released from internal pools affects synaptic DCV mobility, we bath applied a $\mathrm{Ca}^{2+}$ ionophore (4-bromocalcimycin, also called 4-Br-A23187) in $\mathrm{Ca}^{2+}$-free saline containing $0.5 \mathrm{~mm}$ EGTA, a $\mathrm{Ca}^{2+}$ chelator. On application of the $\mathrm{Ca}^{2+}$ ionophore, DCV mobility increased (Fig. $1 A$ ). These data demonstrate that release of $\mathrm{Ca}^{2+}$ from internal $\mathrm{Ca}^{2+}$ pools is sufficient to mobilize DCVs in synaptic boutons.

Because the ER is a potential source of stored $\mathrm{Ca}^{2+}$, we explored the role of $\mathrm{Ca}^{2+}$ derived from ER in DCV mobilization. Specifically, we focused on the RyR, which mediates $\mathrm{Ca}^{2+}$-induced $\mathrm{Ca}^{2+}$ release (CICR) from the ER. Consistent with a potential role for $\mathrm{ER} \mathrm{Ca}^{2+}$ stores, application of the RyR channel opener caffeine in $\mathrm{Ca}^{2+}$-free saline evoked DCV mo- 
A

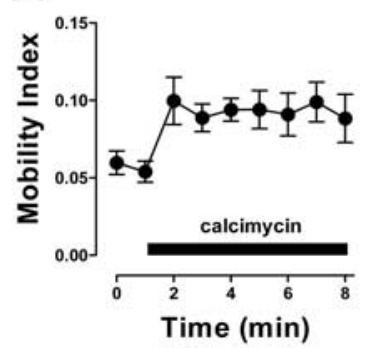

C

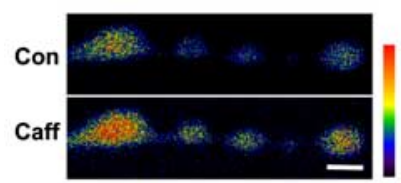

B

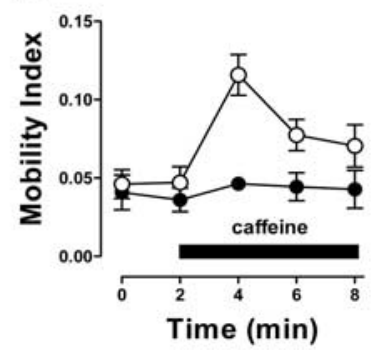

D

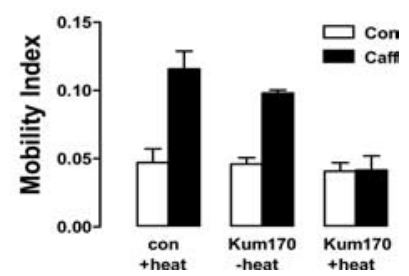

Figure 1. $\mathrm{Ca}^{2+}$ release from internal stores induces DCV mobilization. $\boldsymbol{A}, \mathrm{Ca}^{2+}$ ionophore increases DCV motion in synaptic boutons $(n=4)$. Application of $10 \mu \mathrm{m}$ bromo-calcimycin is indicated by the bar. $\boldsymbol{B}$, Time course of vesicle mobilization induced by $20 \mathrm{~mm}$ caffeine $(n=5$; application indicated by bar) in control $(O)$ and ryanodine $(100 \mu \mathrm{m})$-pretreated $(\bigcirc)$ boutons. C, Fluorescence images of boutons loaded with 0GB-1 before and 1 min after caffeine application. Fluorescence intensity is presented on a pseudo-color scale indicated by the colored bar. Scale bar, $2 \mu \mathrm{m}$. In this case, data were averaged from the first, third, and last boutons (starting from the left). $D, D C V$ mobility before and after caffeine application in control animals exposed to brief heating (con + heat, $n=5$ ) and Kum 170 mutants that were either unheated ( - heat, $n=3$ ) or heated (+ heat, $n=4$ ). $\square$, Control; $\square, 2$ min after caffeine application. Error bars indicate SEM. Con, Control; Caff, caffeine.

bilization (Fig. $1 B$, open circles). Three types of experiments verified that the caffeine effect was mediated by $\mathrm{Ca}^{2+}$ release from the ER. First, treating the preparation with ryanodine at a concentration known to block RyRs prevented DCV mobilization in response to caffeine (Fig. $1 B$, filled circles). Second, the $\mathrm{Ca}^{2+}$ indicator dextran-conjugated OGB-1 revealed that caffeine application in the absence of extracellular $\mathrm{Ca}^{2+}$ increased cytoplasmic $\mathrm{Ca}^{2+}$ in presynaptic boutons (Fig. 1C): the $\mathrm{Ca}^{2+}$ signal increased by $42.8 \pm 7.2 \%(n=6)$. Finally, genetically depleting $\mathrm{ER} \mathrm{Ca}^{2+}$ stores blocked caffeine-evoked vesicle mobilization. For the latter experiments, we used the Ca-P60A ${ }^{\text {Kum170 }}$ (Kum170) mutant, which results in temperature-sensitive loss of function of the $\mathrm{Ca}^{2+}$ pump that maintains ER Ca ${ }^{2+}$ storage (Sanyal et al., 2005). Prolonged paralysis at room temperature is induced in the Kum 170 SERCA mutant by brief exposure to $40^{\circ} \mathrm{C}$. Using this heating protocol, we found that exposing Kum 170 mutant larvae to the restrictive temperature for 8 min blocked DCV mobilization induced by subsequent application of caffeine at room temperature (Fig. 1D). However, DCV mobilization in response to caffeine was evident in unheated Kum170 animals (i.e., under permissive conditions), as well as in control animals exposed to the same heating regimen (Fig. 1D). Hence, disabling SERCA blocked the caffeine-induced increase in DCV motion in synaptic boutons. Together, the effects of the SERCA pump mutant and ryanodine, in addition to cytoplasmic $\mathrm{Ca}^{2+}$ measurements in synaptic boutons, establish that $\mathrm{Ca}^{2+}$ release from ER in the absence of $\mathrm{Ca}^{2+}$ influx from external medium is sufficient to induce DCV mobilization in synaptic boutons.
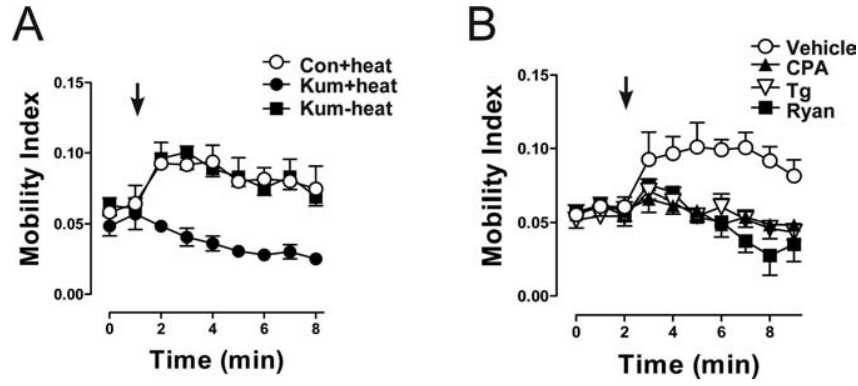

Figure 2. Inhibition of $\mathrm{Ca}^{2+}$ release from ER $\mathrm{Ca}^{2+}$ stores abolishes sustained activityinduced DCV mobilization. $\boldsymbol{A}$, Time course of DCV mobility before and after $15 \mathrm{~s}, 70 \mathrm{~Hz}$ stimulus (indicated by arrow) in heated control larvae (Con + heat; $\bigcirc$ ) and in heated (Kum + heat; $O$ ) and unheated (Kum - heat; $\square$ ) Kum 170 mutants. B, Changes in DCV mobility during $15 \mathrm{~s}, 70 \mathrm{~Hz}$ stimulation in control boutons exposed to vehicle ( $0.1 \%$ DMSO; $\bigcirc ; n=5), 50 \mu \mathrm{M} C \mathrm{CA}(\boldsymbol{\Delta} ; n=$ 5), $20 \mu \mathrm{M} \mathrm{Tg}(\nabla ; n=5)$, or ryanodine (Ryan; $\square ; n=3)$. Error bars indicate SEM.

\section{$\mathrm{ER} \mathrm{Ca}^{2+}$ release is necessary for activity-induced mobilization of DCVs}

Prolonged mobilization of DCVs is induced by brief tetanic stimulation of motor neurons (Shakiryanova et al., 2005). Specifically, stimulation at $70 \mathrm{~Hz}$ for $15 \mathrm{~s}$ induces DCV mobilization for $>6$ min. This effect is evident in Kum170 larvae at permissive temperatures and in control animals briefly heated to $40^{\circ} \mathrm{C}$. However, the tetanus failed to evoke DCV mobilization in Kum170 mutant synaptic boutons after the exposure to $40^{\circ} \mathrm{C}$ (Fig. $2 \mathrm{~A}$ ). The implication of ER $\mathrm{Ca}^{2+}$ stores was verified with the SERCA inhibitor Tg. Boutons were incubated with Tg for $20 \mathrm{~min}$ in the absence of bath $\mathrm{Ca}^{2+}$ according to previous studies with the Drosophila NMJ (Kuromi and Kidokoro, 2002). Then, $\mathrm{Ca}^{2+}$-free saline was replaced with $\mathrm{Ca}^{2+}$-containing saline before stimulation. In contrast to the vehicle control, stimulation-evoked DCV mobilization was abolished in boutons after depletion of ER $\mathrm{Ca}^{2+}$ with Tg (Fig. $2 \mathrm{~B}$ ). Incubation of boutons with CPA, another SERCA inhibitor structurally unrelated and mechanistically distinct from Tg, also prevented activity-induced DCV mobilization (Fig. 2 B). Finally, inhibition of RyRs with ryanodine eliminated DCV mobilization induced by stimulation in the presence of extracellular $\mathrm{Ca}^{2+}$ (Fig. $2 \mathrm{~B}$ ). Thus, genetic and pharmacological experiments show that $\mathrm{Ca}^{2+}$ influx through plasma membrane voltage-gated channels cannot induce sustained DCV mobilization on its own. Instead, $\mathrm{Ca}^{2+}$ influx from the extracellular medium induces RyR-mediated $\mathrm{Ca}^{2+}$ release from the ER that is necessary for activity-induced DCV mobilization.

\section{Activity-induced mobilization requires $\mathrm{Ca}^{2+}$ release in the presynaptic neuron}

Because Drosophila has a single SERCA gene that is expressed in neurons and muscle (Sanyal et al., 2005), the Kum170 mutant and the bath-applied inhibitors affected both presynaptic and postsynaptic SERCA function. The latter could be relevant because there is $\mathrm{Ca}^{2+}$-dependent retrograde signaling at the Drosophila NMJ (Haghighi et al., 2003). However, presynaptic DCV mobilization persists even after release is blocked or postsynaptic receptors are desensitized or inhibited (Shakiryanova et al., 2005) (see Materials and Methods), suggesting that presynaptic signaling is responsible. To test explicitly whether presynaptic $\mathrm{ER} \mathrm{Ca}^{2+}$ release initiates DCV mobilization, SERCA pumps were disabled only in neurons with targeted expression of a conditional dominant-negative SERCA subunit (UAS-Kum170) (Sanyal et al., 2005). This was feasible because this construct and the GFPtagged peptide share the same promoter, which is activated by the 


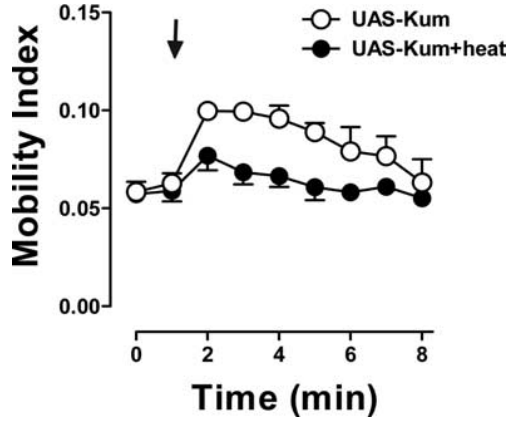

Figure 3. Inhibition of $\mathrm{Ca}^{2+}$ release from presynaptic neuronal but not postsynaptic muscle ER abolishes activity-induced DCV mobilization. The time course of DCV mobility before and after the $15 \mathrm{~s}, 70 \mathrm{~Hz}$ stimulus (indicated by arrow) in unheated larvae $(O)$ and heated $(\mathbf{O})$ larvae expressing a temperature-sensitive dominant-negative SERCA subunit UAS-Kum170 (UAS-Kum) specifically in neurons is shown. Error bars indicate SEM.

transcription factor GAL4. Hence, any GAL4 driver would necessarily induce coexpression of the GFP-tagged peptide and the dominant-negative SERCA subunit. In our case, the panneuronal driver elav-GAL4 was used to produce coexpression in neurons but not in postsynaptic muscle. Under permissive conditions, activity-dependent mobilization was unaffected, but under restrictive conditions (i.e., after exposure to $40^{\circ} \mathrm{C}$ for $8 \mathrm{~min}$ ), the sustained increase in DCV mobility induced by electrical stimulation was abolished (Fig. 3). Thus, activity-dependent DCV mobilization requires $\mathrm{ER} \mathrm{Ca}^{2+}$ release in the presynaptic nerve terminal.

\section{Presynaptic $\mathrm{Ca}^{2+}$ release triggers, but does not sustain, DCV mobilization}

We then addressed whether presynaptic $\mathrm{Ca}^{2+}$ sustains prolonged mobilization. Specifically, to test whether DCV mobilization is maintained by residual $\left[\mathrm{Ca}^{2+}\right]_{\mathrm{i}}$ after stimulation, the $\left[\mathrm{Ca}^{2+}\right]_{\mathrm{i}}$ time course in synaptic boutons was determined with $\mathrm{Ca}^{2+}$ indicators. Notably, previous studies had shown that brief bouts of activity result in transient elevation of $\mathrm{Ca}^{2+}$ but long bouts of stimulation can produce a prolonged $\mathrm{Ca}^{2+}$ response (Kuromi and Kidokoro, 2002; Macleod et al., 2002; Sanyal et al., 2005). Therefore, motor neuron terminals were loaded with dextranconjugated OGB-1 and stimulated at $70 \mathrm{~Hz}$ for $15 \mathrm{~s}$ [i.e., the stimulus for maximal synaptic DCV mobilization (Shakiryanova et al., 2005)]. This activity caused a marked enhancement of OGB-1 fluorescence (Fig. 4A,B). After cessation of stimulation, presynaptic $\left[\mathrm{Ca}^{2+}\right]_{\mathrm{i}}$ decayed back to baseline levels within $10-15$ $\mathrm{s}$ (Fig. $4 B$ ). Thus, the elevation in cytoplasmic $\mathrm{Ca}^{2+}$ is far more transient than the many minutes of vesicle mobilization induced by this stimulus (Fig. 2).

Because dextran-conjugated OGB-1 was loaded into synaptic boutons via cut axons (see Materials and Methods), we considered whether this procedure could have affected the $\mathrm{Ca}^{2+}$ time course. To address this concern, $\mathrm{Ca}^{2+}$ imaging experiments were performed with transgenic animals expressing the cytoplasmic $\mathrm{Ca}^{2+}$ sensor protein Cameleon 2.1 (Dieglemann et al., 2002). Ratiometric Cameleon measurements are based on fluorescence resonance energy transfer between ECFP and EYFP, resulting in an increased EYFP emission at $535 \mathrm{~nm}$ and a corresponding decrease in ECFP emission at $485 \mathrm{~nm}$ in the presence of elevated $\left[\mathrm{Ca}^{2+}\right]_{\mathrm{i}}$. Recordings of the EYFP and ECFP emission intensities in boutons of intact motor neurons revealed opposing ECFP and EYFP signal changes evoked by $15 \mathrm{~s}$ of stimulation at $70 \mathrm{~Hz}$ (Fig. $4 C)$. Importantly, the normalized EYFP/ECFP ratio increased
A

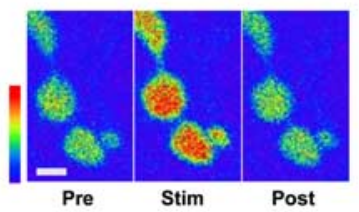

C

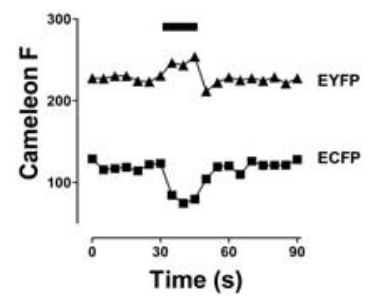

B

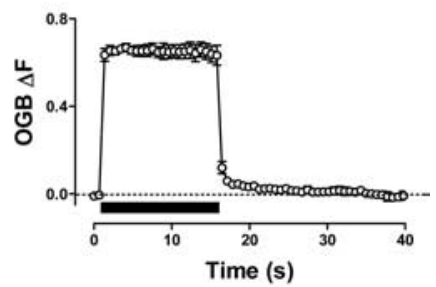

D

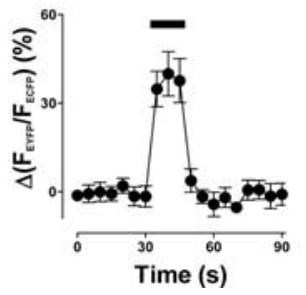

Figure 4. Activity-evoked elevation in presynaptic $\left[\mathrm{Ca}^{2+}\right]_{\mathrm{i}}$ is transient. $\boldsymbol{A}, 0 \mathrm{~GB}$ fluorescence in boutons before (Pre), during (Stim), and after (Post) $15 \mathrm{~s}, 70 \mathrm{~Hz}$ stimulation. Scale bar, $2 \mu \mathrm{m}$. In this example, data were averaged from the two large round boutons. $B$, Relative changes in $\left[\mathrm{Ca}^{2+}\right]_{\mathrm{i}}$ during $15 \mathrm{~s}, 70 \mathrm{~Hz}$ stimulation (indicated by bar) in $0 \mathrm{~GB}$-1-loaded boutons $(n=4)$. The dashed line shows the resting level of $\left[\mathrm{Ca}^{2+}\right]_{\mathrm{i}}$. C, Consecutive recordings of the Cameleon EYFP

$(\mathbf{\Delta})$ and ECFP ( $\square$ ) emission intensities during $15 \mathrm{~s}, 70 \mathrm{~Hz}$ stimulation in boutons expressing Cameleon 2.1. D, Percentage change in the EYFP/ECFP ratio during $15 \mathrm{~s}, 70 \mathrm{~Hz}$ stimulation indicated by the bar $(n=7)$. The EYFP/ECFP ratio was normalized to the ratio before electrical stimulation. Error bars indicate SEM.

during stimulation and returned rapidly to the resting level after the end of stimulation (Fig. 4D). The time course of the $\mathrm{Ca}^{2+}$ response revealed by Cameleon agrees with data obtained from boutons loaded with OGB-1. Thus, both chemical and transgenic protein indicators show that residual $\mathrm{Ca}^{2+}$ is too transient to maintain the long-lasting DCV mobilization induced by a brief tetanus. This implies that presynaptic $\mathrm{Ca}^{2+}$ release triggers, but does not sustain, prolonged mobilization.

\section{CaMKII is required for activity-induced mobilization}

The mismatch in the time courses of $\left[\mathrm{Ca}^{2+}\right]_{\mathrm{i}}$ and mobilization suggests that downstream signaling sustains activity-induced DCV mobilization. CaMKII is an established long-acting mediator of transient $\mathrm{Ca}^{2+}$ changes (see Discussion). Therefore, we focused on this signaling enzyme. First, we genetically targeted expression of the CaMKII inhibitory Ala peptide (Griffith et al., 1994) to neurons, but this yielded uninterpretable results: activity-dependent mobilization was reduced, but baseline DCV motion was increased (data not shown). This might reflect a developmental effect that is a consequence of the presynaptic hyperexcitability and incomplete CaMKII inhibition induced by genetic expression of CaMKII inhibitory peptides (Griffith et al., 1994). Therefore, we used two pharmacological approaches to ensure acute and robust inhibition of CaMKII without any potential for long-term developmental effects.

First, boutons were treated before stimulation with the CaMKII inhibitors KN-62 or KN-93, which bind to the calmodulin site on the kinase. In both cases, sustained DCV mobilization in response to a tetanus was abolished (Fig. 5A). However, KN92, an inactive analog of KN-93, did not affect vesicle mobilization. Second, CaMKII activity was specifically inhibited with the membrane-permeant version (i.e., Antennapedia peptide fused) of AIP-II, which binds to the substrate site of CaMKII. Exposure of boutons to AIP-II eliminated activity-induced mobilization of 

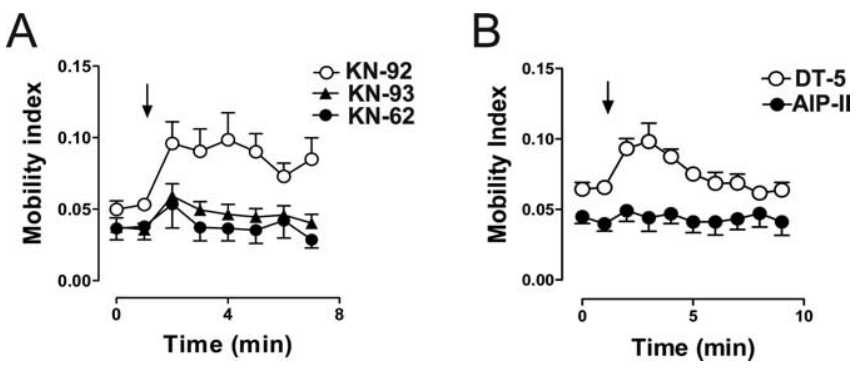

Figure 5. Inhibition of CaMKII eliminates activity-induced DCV mobilization. $A$, Time course of DCV mobility before and after $15 \mathrm{~s}, 70 \mathrm{~Hz}$ stimulation in boutons preincubated with $\mathrm{KN}-92$ $(\bigcirc ; n=8), \mathrm{KN}-93(\mathbf{\Delta} ; n=9)$, or KN-62 $(\mathbf{O} ; n=5)$. Compounds were applied $15 \mathrm{~min}$ before stimulation at $10 \mu \mathrm{m}$. $\boldsymbol{B}$, Changes in DCV mobility before and after $15 \mathrm{~s}, 70 \mathrm{~Hz}$ stimulation in boutons treated with control peptide (DT-5; $\bigcirc ; n=6$ ) or the CaMKII inhibitory peptide AIP-II $(\bullet ; n=5)$. Peptides were applied $1 \mathrm{~h}$ before stimulation at $10 \mu \mathrm{m}$. Arrows indicate stimulation points. Error bars indicate SEM.

DCVs (Fig. 5B). In contrast, DCV mobilization in response to stimulation was evident in boutons treated with the control peptide DT-5. Mobilization was shorter-lived in the latter case than in other experiments, but this could be attributable to the $1 \mathrm{~h}$ preincubation in this experiment or a nonspecific effect of membrane-permeant peptides. Importantly, this kinetic change does not change the main conclusion: structurally and mechanistically distinct specific inhibitors show that CaMKII activation is required for activity-induced DCV mobilization.

\section{CaMKII is a downstream effector of RyR-mediated $\mathrm{Ca}^{2+}$ release}

Our experimental findings indicate that both $\mathrm{Ca}^{2+}$ release via RyRs and CaMKII activation are necessary for activity-induced DCV mobilization. CaMKII might function either as a downstream effector of released $\mathrm{ER} \mathrm{Ca}^{2+}$ or as an upstream activator of RyRs to prime CICR at nerve terminals. In the latter case, the requirement for CaMKII could be bypassed by evoking $\mathrm{Ca}^{2+}$ release from internal stores without the involvement of RyRs. In contrast, if CaMKII is a downstream effector of released $\mathrm{Ca}^{2+}$, then inhibition of CaMKII should disrupt DCV mobilization regardless of the mechanism underlying the release of stored intracellular $\mathrm{Ca}^{2+}$.

To distinguish between these possibilities, we studied the action of veratridine, a sodium channel opener that increases $\left[\mathrm{Ca}^{2+}\right]_{\mathrm{i}}$ in Drosophila nerve terminals in the absence of external $\mathrm{Ca}^{2+}$ (Kuromi and Kidokoro, 2002). Cytoplasmic sodium is known to induce $\mathrm{Ca}^{2+}$ release from nerve-terminal mitochondria (Yang et al., 2003). Therefore, we first examined whether veratridine acts independently of the ER. Imaging verified that 10 $\mu \mathrm{M}$ veratridine increases cytoplasmic $\mathrm{Ca}^{2+}$ : the OGB-1 signal increased by $50 \pm 17 \%(n=4)$ in the absence of bath $\mathrm{Ca}^{2+}$. Veratridine also induced DCV mobilization in the absence of bath $\mathrm{Ca}^{2+}$. However, in contrast to the effects of caffeine and activity, the increase in DCV mobility induced by veratridine persisted even after depleting ER $\mathrm{Ca}^{2+}$ stores by heating mutant SERCA pump larvae (Fig. 6A). This implies that veratridine does not rely on $\mathrm{ER} \mathrm{Ca}^{2+}$ stores to induce mobilization and so bypasses RyRs. Despite the lack of involvement of RyRs, mobilization by veratridine required CaMKII activity: it was inhibited by KN-93 but unaffected by inert KN-92 (Fig. 6B). This cannot be attributed to a coincidental requirement for CaMKII for the action of veratridine because inhibition of CaMKII also prevented DCV mobilization in response to a $\mathrm{Ca}^{2+}$ ionophore (Fig. 6C). In contrast, the inactive analog KN-92 did not affect mobilization of
DCVs induced by the ionophore. Because RyR-independent $\mathrm{Ca}^{2+}$ release can only induce DCV mobilization when CaMKII is active, CaMKII is required as a downstream effector of released internal $\mathrm{Ca}^{2+}$.

This conclusion is also supported by $\mathrm{Ca}^{2+}$ imaging. If CaMKII induced mobilization by activating RyRs, then inhibiting CaMKII should reduce the $\mathrm{Ca}^{2+}$ response evoked by a tetanus. However, the increase in presynaptic $\mathrm{Ca}^{2+}$ in response to a tetanus and the subsequent decay in residual $\left[\mathrm{Ca}^{2+}\right]_{\mathrm{i}}$ are not affected by CaMKII inhibition (Fig. 7). Hence, activity-evoked DCV mobilization is not limited by CaMKII-dependent RyR activity but occurs as a result of CaMKII that was stimulated by RyR-mediated $\mathrm{Ca}^{2+}$ release.

\section{PTP of neuropeptide secretion requires the RyR and CaMKII} Increased DCV mobility induced by a brief high-frequency conditioning tetanus is correlated with PTP of neuropeptide secretion (Shakiryanova et al., 2005). Specifically, two $30 \mathrm{~s}$ bouts of 3 $\mathrm{Hz}$ stimulation that do not induce mobilization evoke reproducible neuropeptide secretion responses, but $15 \mathrm{~s}$ of $70 \mathrm{~Hz}$ stimulation, which induces mobilization (Fig. 2), increases neuropeptide release evoked $2.5 \mathrm{~min}$ later by low-frequency activity. Although this PTP of neuropeptide release occurs when mobilization is at its peak, it is not known whether inhibiting DCV mobilization affects this synaptic plasticity.

To test whether inhibiting mobilization-inducing signaling alters PTP, nerve terminals were treated with KN-93, ryanodine, or their associated controls (KN-92 or vehicle, respectively) and stimulated according to above mentioned protocol. PTP of neuropeptide release was evident in the controls (Fig. 8, left column). However, no PTP was induced after inhibiting CaMKII (Fig. $8 \mathrm{~A}$, right). Likewise, inhibition of RyRs blocked PTP (Fig. $8 B$, right). Therefore, CaMKII activated by RyR-mediated CICR from the ER induces DCV mobilization in synaptic boutons and PTP of neuropeptide secretion.

\section{Discussion}

It has long been speculated that vesicle mobility is increased to facilitate secretion. In favor of this model, in vitro studies showed that DCV mobility limits neuropeptide release (Burke et al., 1997; Han et al., 1999), and pharmacologically increasing DCV mobility enhances neuropeptide release ( $\mathrm{Ng}$ et al., 2002, 2003). However, native signaling mechanisms that induce such DCV mobilization in a nerve terminal in vivo and the impact of this signaling on release have not been determined. Indeed, the importance of vesicle mobility has been obscured because the term mobilization has been co-opted to include almost any process that increases release. The recent direct detection of regulated vesicle mobility in motor neuron terminals has opened the door to probing the signaling involved in bona fide vesicle mobilization and its connection to synaptic plasticity. Specifically, we reasoned that identifying the signaling that controls vesicle motion would serve two purposes: (1) to delineate regulatory mechanisms in the nerve terminal and (2) to produce the opportunity to test whether inhibiting mobilization affects release for the first time. Here, synaptic DCV motion and neuropeptide release were imaged in vivo to show that both activity-dependent increases in DCV mobility and PTP require presynaptic ER $\mathrm{Ca}^{2+}$ release via RyRs leading to CaMKII activation. The use of a single signaling pathway for controlling DCV motion and release provides the first empirical indication of a mechanistic connection between mobilization and synaptic plasticity. Baseline DCV mobility and secretion evoked by low-frequency activity are not markedly affected by 
inhibiting RyRs or CaMKII (Figs. 2, 5, 8). Therefore, this signaling pathway has a minor role until it is recruited by tetanic activity. The activity requirement for recruitment of RyR-CaMKII-activated mobilization and PTP is well matched to the native activity found at the larval NMJ [i.e., rhythmic bursting (Klose et al., 2005)] and the activity patterns known to be optimal for neuropeptide release. Given past in vitro studies of DCV mobility (Burke et al., 1997; Han et al., 1999; Ng et al., 2002, 2003), the in vivo results presented here are consistent with the conclusion that physiological activity triggers RyR-activated CaMKII to increase DCV mobility, which in turn contributes to PTP of neuropeptide secretion.

The necessity for RyR-mediated $\mathrm{Ca}^{2+}$ release for mobilization and PTP is surprising because bulk $\mathrm{Ca}^{2+}$ is effectively elevated in Drosophila motor neuron terminals by $\mathrm{Ca}^{2+}$ influx through plasma membrane voltage-gated channels even when $\mathrm{ER} \mathrm{Ca}^{2+}$ is depleted (Kuromi and Kidokoro, 2002; Sanyal et al., 2005). Indeed, as noted above, plasma membrane voltage-gated $\mathrm{Ca}^{2+}$ channels are sufficient for triggering some neuropeptide release at low levels of activity but apparently cannot induce sustained mobilization without the participation of RyRs. Thus, as far as mobilization is concerned, CaMKII is apparently more sensitive to $\mathrm{Ca}^{2+}$ released from intracellular stores than extracellular $\mathrm{Ca}^{2+}$ influx. Perhaps this specificity arises because of positioning of CaMKII closer to RyRs than voltage-gated channels in the presynaptic plasma membrane. Alternatively, a small amount of $\mathrm{Ca}^{2+}$ influx through a voltage-gated channel may trigger a much larger $\mathrm{Ca}^{2+}$ spark by opening multiple RyRs. This could be amplified by production of RyR-mediated $\mathrm{Ca}^{2+}$ waves. Thus, local $\mathrm{Ca}^{2+}$ elevation at the surface, which is sufficient for triggering exocytosis of docked vesicles, could give rise to a propagating CICR from the ER to activate CaMKII in the cytoplasm where most DCVs reside. This positive feedback might also set the threshold activity required for activating CaMKII-induced mobilization. Hence, RyRs could control the activity dependence and spatial propagation of vesicle mobilization. Regardless of the underlying mechanism, the requirement for $\mathrm{ER} \mathrm{Ca}^{2+}$ stores delineated here implies that the $\mathrm{Ca}^{2+}$ sources for triggering exocytosis (i.e., plasma membrane voltage-gated $\mathrm{Ca}^{2+}$ channels) and sustained mobilization (i.e., intracellular RyRs) are distinct.

Our studies establish that RyR-CaMKII-dependent DCV mobilization is initiated in the presynaptic terminal. First, mobilization is blocked by disabling neuronal SERCA function, while leaving muscle SERCA intact (Fig. 3). Second, the activityinduced increase in presynaptic DCV motion does not depend on standard synaptic transmission between nerve and muscle: mobilization persists after inhibiting exocytosis (Shakiryanova et al., 2005) or postsynaptic glutamate receptors with desensitizing doses of glutamate (Shakiryanova et al., 2005) or an antagonist (see Materials and Methods). RyR and CaMKII are present in muscle as well as neurons (Hasan and Rosbash, 1992; Griffith et al., 1994; Haghighi et al., 2003; Lu et al., 2003), but it is not known whether muscle CaMKII, which participates in retrograde signaling (Haghighi et al., 2003), is activated by $\mathrm{Ca}^{2+}$ flux through RyRs. Further study may show that the intimate functional relationship between the RyR and CaMKII exists on both sides of the synapse.

We also discovered that a short-lived elevation in $\mathrm{Ca}^{2+}$ in-
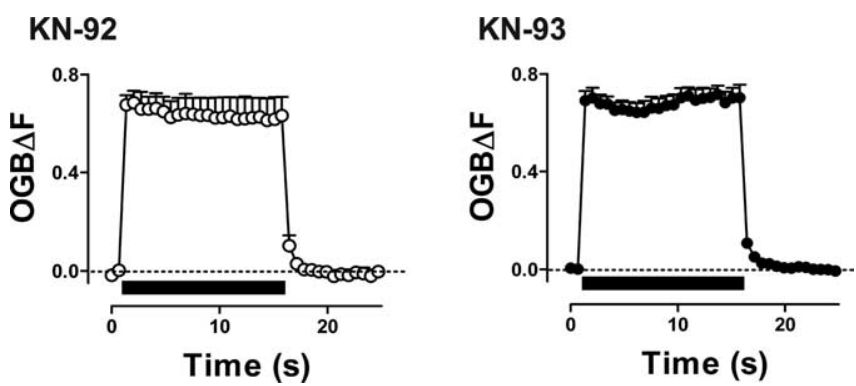

Figure 7. Presynaptic $\left[\mathrm{Ca}^{2+}\right]_{\mathrm{i}}$ is not affected by inhibition of CaMKII. Changes in OGB-1 fluorescence intensities in boutons treated with KN-92 $(n=5)$ and KN-93 $(n=5)$ in response to $15 \mathrm{~s}, 70 \mathrm{~Hz}$ stimulation (indicated by bars).

duces long-lasting mobilization and PTP. Hence, residual $\mathrm{Ca}^{2+}$, which is important for synaptic plasticity (Zucker, 1999), does not sustain these responses. The downstream involvement of CaMKII suggests that phosphorylation leading to a sustained increase in vesicle mobility and secretion persists after residual $\mathrm{Ca}^{2+}$ has dissipated. This could reflect either slow substrate dephosphorylation because of limited phosphatase activity or prolonged activation of CaMKII, possibly by autophosphorylation on T287 of the Drosophila CaMKII (Wang et al., 1998; Lu et al., 2003). The presence of some autophosphorylated CaMKII at the resting NMJ (Hodge et al., 2006) might also explain the small reduction in DCV mobility induced by CaMKII inhibitors applied at rest (Fig. 5). Unfortunately, because it is very difficult to specifically measure presynaptic autophosphorylation of CaMKII with phosphospecific antibodies in the context of far greater amounts of CaMKII in the postsynaptic side of the Drosophila NMJ (Hodge et al., 2006; L. Griffith, personal communication), the prevalence of presynaptic autophosphorylated CaMKII is not known. Likewise, we cannot exclude the participation of other $\mathrm{Ca}^{2+}$-signaling proteins. Nevertheless, it is clear that CaMKII-induced phosphorylation is critical for sustained DCV mobilization and PTP after $\mathrm{Ca}^{2+}$ has returned to baseline levels.

Our results also hint that the RyR-CaMKII pathway may not be the sole signaling mechanism for regulating DCV mobility in synaptic boutons. First, inhibiting $\mathrm{Ca}^{2+}$ release revealed a sustained small decrease in DCV mobility (Fig. 2), which was not detected in the absence of $\mathrm{Ca}^{2+}$ influx (Shakiryanova et al., 2005). Previously, we showed that DCV mobilization in type Ib boutons begins to reverse after $\sim 5 \mathrm{~min}$ (Shakiryanova et al., 2005). Given that CaMKII is required for sustained mobilization, we hypothesize that dephosphorylation resets DCV dynamics. Future experiments could explore whether an activity-sensitive 
A
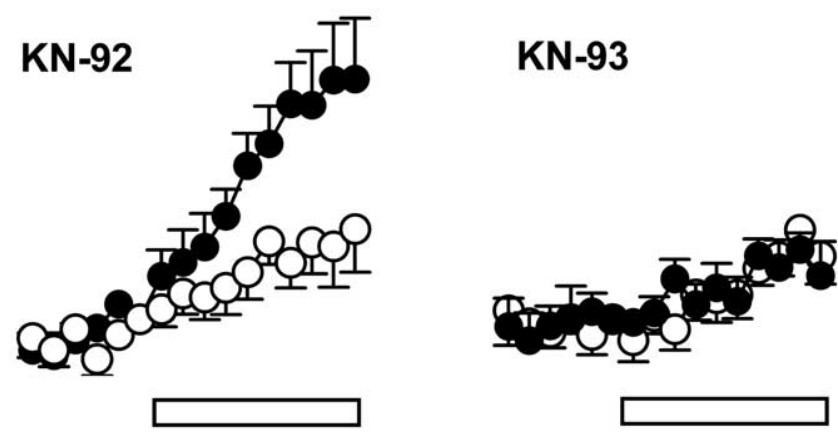

B

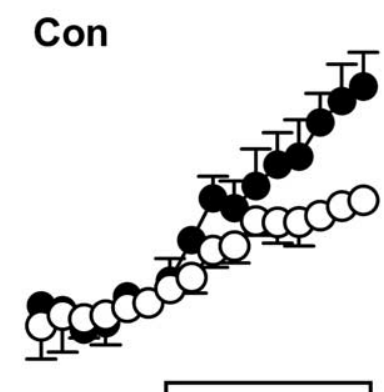

Con

Ryan

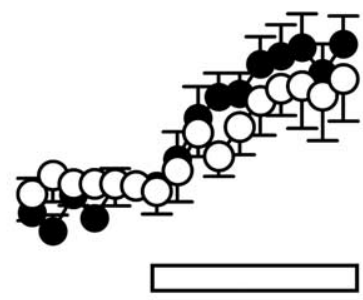

$1 \%$ Release

Figure 8. PTP of neuropeptide release requires CaMKII and the RyR. Release responses to $30 \mathrm{~s}$ of $3 \mathrm{~Hz}$ stimulation before and after a $15 \mathrm{~s}, 70 \mathrm{~Hz}$ conditioning tetanus in boutons treated with KN-92 $(n=9)$ or KN-93 $(n=7)(\boldsymbol{A})$ and vehicle (Con; $n=6)$ or ryanodine (Ryan; $n=6)$ $(\boldsymbol{B}) . \bigcirc$, Release before conditioning stimulus; $\boldsymbol{\Theta}, 2.5 \mathrm{~min}$ after conditioning tetanus. Open bars indicate $3 \mathrm{~Hz}$ stimulation. Release was measured as loss of peptide fluorescence. Error bars indicate SEM.

phosphatase such as calcineurin reduces DCV mobility. Such a mechanism could be responsible for reducing steady-state phosphorylation in the absence of $\mathrm{Ca}^{2+}$ release and reversing the effects of additional CaMKII-mediated phosphorylation when RyRs are active. We also observed that there was a small transient increase in DCV motion in many of the experiments in which RyRs and CaMKII were inhibited. The simplest explanation for this result is that this is a consequence of incomplete inhibition of mobilization combined with the above described demobilization mechanism. However, we have not excluded that this represents the direct, but inefficient, activation of CaMKII by $\mathrm{Ca}^{2+}$ influx. It would be interesting to test whether DCV mobility is transient and limited to near the cell surface when RyRs are inhibited, but this is beyond the resolution of current methods that can be used with the intact living NMJ.

The signaling responsible for increasing DCV mobility and PTP of neuropeptide secretion in Drosophila motor neuron terminals may be widely relevant. For example, this pathway could be responsible for mobilization in neuroendocrine cells (Allersma et al., 2006). Likewise, CaMKII-mediated DCV mobilization may contribute to the increase in activity-dependent

neuropeptide release by dendritic spines (Lochner et al., 2006) induced by release of ER $\mathrm{Ca}^{2+}$ (Ludwig et al., 2002). The signaling described here could also be important in synapses capable of SSV mobilization (e.g., the NMJ), which shares many features with DCV mobilization (see Introduction). In fact, presynaptic RyR and CaMKII contribute to synaptic potentiation in Aplysia synapses and the neurotrophin-induced increase in spontaneous neurotransmitter release in developing NMJs (Jin and Hawkins, 2003; He et al., 2000). SSV mobility studies should be able to test whether mobilization occurs and is necessary in these cases of synaptic plasticity.

\section{References}

Allersma MW, Bittner MA, Axelrod D, Holz RW (2006) Motion matters: granule motion adjacent to the plasma membrane and exocytosis. Mol Biol Cell 17:2424-2438.

Burke NV, Han W, Li D, Takimoto K, Watkins SC, Levitan ES (1997) Neuronal peptide release is limited by secretory granule mobility. Neuron 19:1095-1102.

Diegelmann S, Fiala A, Leibold C, Spall T, Buchner E (2002) Transgenic flies expressing the fluorescence calcium sensor Cameleon 2.1 under UAS control. Genesis 34:95-98.

Gaffield MA, Rizzoli SO, Betz WJ (2006) Mobility of synaptic vesicles in different pools in resting and stimulated frog motor nerve terminals. Neuron 51:317-325.

Griffith LC, Wang J, Zhong Y, Wu CF, Greenspan RJ (1994) Calcium/ calmodulin-dependent protein kinase II and potassium channel subunit eag similarly affect plasticity in Drosophila. Proc Natl Acad Sci USA 91:10044-10048.

Haghighi AP, McCabe BD, Fetter RD, Palmer JE, Hom S, Goodman CS (2003) Retrograde control of synaptic transmission by postsynaptic CaMKII at the Drosophila neuromuscular junction. Neuron 39:255-267.

Han W, Ng YK, Axelrod D, Levitan ES (1999) Neuropeptide release by efficient recruitment of diffusing cytoplasmic secretory vesicles. Proc Natl Acad Sci USA 96:14577-14582.

Hasan G, Rosbash M (1992) Drosophila homologs of two mammalian intracellular $\mathrm{Ca}^{2+}$-release channels: identification and expression patterns of the inositol 1,4,5-triphosphate and the ryanodine receptor genes. Development 116:967-975.

He X, Yang F, Xie Z, Lu B (2000) Intracellular $\mathrm{Ca}^{2+}$ and $\mathrm{Ca}^{2+} /$ calmodulindependent kinase II mediate acute potentiation of neurotransmitter release by neurotrophin-3. J Cell Biol 149:783-792.

Heifetz Y, Wolfner MF (2004) Mating, seminal fluid components, and sperm cause changes in vesicle release in the Drosophila female reproductive tract. Proc Natl Acad Sci USA 101:6261-6266.

Hodge JJ, Mullasseril P, Griffith LC (2006) Activity-dependent gating of CaMKII autonomous activity by Drosophila CASK. Neuron 51:327-337.

Husain QM, Ewer J (2004) Use of targetable GFP-tagged neuropeptide for visualizing neuropeptide release following execution of a behavior. J Neurobiol 59:181-191.

Jin I, Hawkins RD (2003) Presynaptic and postsynaptic mechanisms of a novel form of homosynaptic potentiation at Aplysia sensory motor neurons. J Neurosci 23:7288-7297.

Klose MK, Chu D, Xiao C, Seroude L, Robertson RM (2005) Heat shockmediated thermoprotection of larval locomotion compromised by ubiquitous overexpression of Hsp70 in Drosophila melanogaster. J Neurophysiol 94:3563-3572.

Kula E, Levitan ES, Pyza E, Rosbash M (2006) PDF cycling in the dorsal protocerebrum of the Drosophila brain is not necessary for circadian clock function. J Biol Rhythms 21:104-117.

Kuromi H, Kidokoro Y (2002) Selective replenishment of two vesicle pools depends on the source of $\mathrm{Ca}^{2+}$ at the Drosophila synapse. Neuron 35:333-343.

Lemke EA, Klingauf J (2005) Single synaptic vesicle tracking in individual hippocampal boutons at rest and during synaptic activity. J Neurosci 25:11034-11044.

Levitan ES, Lanni F, Shakiryanova D (2007) In vivo imaging of vesicle motion and release at the Drosophila neuromuscular junction. Nat Protoc 2:1117-1125.

Lochner JE, Honigman LS, Grant WF, Gessford SK, Hansen AB, Silverman MA, Scalettar BA (2006) Activity-dependent release of tissue plasmino- 
gen activator from the dendritic spines of hippocampal neurons revealed by live-cell imaging. J Neurobiol 66:564-577.

Lu CS, Hodge JJ, Mehren J, Sun XX, Griffith LC (2003) Regulation of the $\mathrm{Ca}^{2+} / \mathrm{CaM}$-responsive pool of CaMKII by scaffold-dependent autophosphorylation. Neuron 40:1185-1197.

Ludwig M, Sabatier N, Bull PM, Landgraf R, Dayanithi G, Leng G (2002) Intracellular calcium stores regulate activity-dependent neuropeptide release from dendrites. Nature 418:85-89.

Macleod GT, Hegstroem-Wojtowicz M, Charlton MP, Atwood HL (2002) Fast calcium signals in Drosophila nerve terminals. J Neurophysiol 88:2659-2663.

Ng YK, Lu X, Levitan ES (2002) Physical mobilization of secretory vesicles facilitates neuropeptide release by nerve growth factor-differentiated PC12 cells. J Physiol (Lond) 542:395-402.

Ng YK, Lu X, Gulacsi A, Han W, Saxton MJ, Levitan ES (2003) Unexpected mobility variation among individual secretory vesicles produces an apparent refractory neuropeptide pool. Biophys J 84:4127-4134.

Nunes P, Haines N, Kuppuswamy V, Fleet DJ, Stewart BA (2006) Synaptic vesicle mobility and presynaptic F-actin are disrupted in a N-ethylmaleimide-sensitive factor allele of Drosophila. Mol Biol Cell 17:4709-4719.

Rao S, Lang C, Levitan ES, Deitcher DL (2001) Visualization of neuropeptide expression, transport, and exocytosis in Drosophila melanogaster. J Neurobiol 49:159-172.

Rea R, Li J, Dharia A, Levitan ES, Sterling P, Kramer RH (2004) Streamlined synaptic vesicle cycle in cone photoreceptor terminals. Neuron 41:755-766.
Sanyal S, Consoulas C, Kuromi H, Basole A, Mukai L, Kidokoro Y, Krishnan KS, Ramaswami M (2005) Analysis of conditional paralytic mutants in Drosophila sarco-endoplasmic reticulum calcium ATPase reveals novel mechanisms for regulating membrane excitability. Genetics 169:737-750.

Scalettar BA (2006) How neurosecretory vesicles release their cargo. Neuroscientist 12:164-176.

Shakiryanova D, Tully A, Hewes RS, Deitcher DL, Levitan ES (2005) Activity-dependent liberation of synaptic neuropeptide vesicles. Nat Neurosci 8:173-178

Shakiryanova D, Tully A, Levitan ES (2006) Activity-dependent synaptic capture of transiting peptidergic vesicles. Nat Neurosci 9:896-900.

Silverman MA, Johnson S, Gurkins D, Farmer M, Lochner JE, Rosa P, Scalettar BA (2005) Mechanisms of transport and exocytosis of dense-core granules containing tissue plasminogen activator in developing hippocampal neurons. J Neurosci 25:3095-3106.

Tokuoka H, Goda Y (2006) Myosin light chain kinase is not a regulator of synaptic vesicle trafficking during repetitive exocytosis in cultured hippocampal neurons. J Neurosci 26: 11606-11614.

Wang Z, Palmer G, Griffith LC (1998) Regulation of Drosophila calcium/ calmodulin-dependent protein kinase II by autophosphorylation analysed by site-directed mutagenesis. J Neurochem 71:378-387.

Yang F, He XP, Russell J, Lu B (2003) $\mathrm{Ca}^{2+}$ influx-independent synaptic potentiation mediated by mitochondrial $\mathrm{Na}^{+}-\mathrm{Ca}^{2+}$ exchanger and protein kinase C. J Cell Biol 163:511-523.

Zucker RS (1999) Calcium- and activity-dependent synaptic plasticity. Curr Opin Neurobiol 9:305-313. 